\title{
Understanding 802.11e Voice Behaviour via Testbed Measurements and Modeling
}

\author{
Ian Dangerfield, David Malone, Douglas J. Leith
}

\begin{abstract}
Analytic modeling and simulation of 802.11 and 802.11e have flagged up a number of potential performance problems with voice in an infrastructure mode network. This body of work has also predicted a number of possible solutions, such as the use of TXOP. In this paper we reproduce a number of these problems in a 802.11 testbed, and demonstrate that analytic models usefully bound performance. We study the impact of buffer size, which is difficult to fully capture in analytic models. We observe an asymmetry in buffer requirements between stations and the access point and explore how buffering and the use of TXOP impact throughput and delay of voice.
\end{abstract}

Keywords- 802.11e, CSMA/CA, buffering, test-bed, voice, measurement

\section{INTRODUCTION}

The 802.11 e MAC protocol extends the standard 802.11 CSMA/CA contention mechanism by allowing the adjustment of MAC parameters that were previously fixed. While the 802.11 e protocol has been extensively studied in the literature, this work is almost entirely confined to analytical and simulation studies. Owing to the lack of available hardware, there have been few experimental studies evaluating the performance of the new 802.11e protocol. Hardware is, however, now available which allows us to investigate 802.11e EDCA operation in a real testing environment. We have constructed an $802.11 \mathrm{e}$ hardware testbed network and in this paper our aim is make use of this testbed to perform experimental measurement and validation of $802.11 \mathrm{e}$ operation.

As a first step, in [1] we compared our expectations (from theory and simulation) with the throughput behaviour of an actual $802.11 \mathrm{e}$ implementation. There is an extensive literature containing simulation [2], [3] and analytic [4], [5] studies/comparisons of the 802.11 and $802.11 \mathrm{e}$ MAC mechanisms. A number of experimental studies, albeit in the context of 802.11 rather than 802.11 e, also show a gap between theoretical predictions and practical performance [6], [7]. In [8] we developed a technique for measuring MAC level delays in our $802.11 \mathrm{e}$ testbed and verified the use of 802.11 e to protect a single delay sensitive flow from saturated data flows.

In [1] we also considered the impact of 802.11 causing the access point (AP) to be a bottle neck for data traffic. This is also known to be a limiting factor for the number of voice calls a network can support [9], [10]. Modelling and simulation has suggested that by prioritising the AP voice capacity can be increased. In this paper we implement these schemes in a test bed and compare the experimental

Work supported by Science Foundation Ireland grant IN3/03/I346. The authors are with the Hamilton Institute, National University of Ireland, Maynooth, Co. Kildare, Ireland. measurements with model predictions, where possible. We also look at the impact of varying the buffer space available, an effect that is difficult to capture in analytic modeling, but has received some simulation based study [11]. Some modeling work has also confirmed that increased buffering alone is not a solution to voice unfairness [12].

In this paper the 802.11 e parameters $C W_{\min }$ and $T X O P$ will be of interest. $C W_{\min }$ is the minimum contention window and is adjustable in powers of two i.e. as $2^{k}$ with integer $k$. TXOP how long a station may transmit when it wins a transmission opportunity. It is a length of time, specified in microseconds.

\section{Testbed Setup}

The $802.11 \mathrm{e}$ wireless testbed is configured in infrastructure mode. It consists of a desktop PC acting as an access point, 18 PC-based embedded Linux boxes based on the Soekris net4801 [13] and one desktop PC acting as client stations. The PC acting as a client records delay measurements for each of its packets, but otherwise behaves as an ordinary client station. All systems are equipped with an Atheros $802.11 \mathrm{~b} / \mathrm{g}$ PCI card with an external antenna. The system hardware configuration is summarised in Table I. All nodes, including the AP, use a Linux 2.6.8.1 kernel and a version of the MADWiFi [14] wireless driver modified to allow us to adjust the $802.11 \mathrm{e} C W_{\min }$, and TXOP parameters. All of the systems are also equipped with a $100 \mathrm{Mbps}$ wired Ethernet port, which is used for control of the testbed from a PC. Specific vendor features on the wireless card, such as turbo mode, are disabled. All of the tests are performed using the $802.11 \mathrm{~b}$ physical maximal transmission rate of $11 \mathrm{Mbps}$ with RTS/CTS disabled and the channel number explicitly set. Since the wireless stations are based on low power embedded systems, we have tested these wireless stations to confirm that the hardware performance (especially the CPU) is not a bottleneck for wireless transmissions at the $11 \mathrm{Mbps} \mathrm{PHY}$ rate used. As noted above, a desktop PC is used as a client to record the per-packet delay measurements. This is to ensure that there is ample disk space, RAM and CPU resources available so that collection of statistics not impact on the transmission of packets.

The configuration of the various network buffers and MAC parameters is detailed in Table II. As we will be interested in the impact of buffering, we have varied the length of certain queues.

The testbed was calibrated as described in [1] by adjusting the positions of stations and antennae until the throughputs achieved by all stations were roughly similar. 


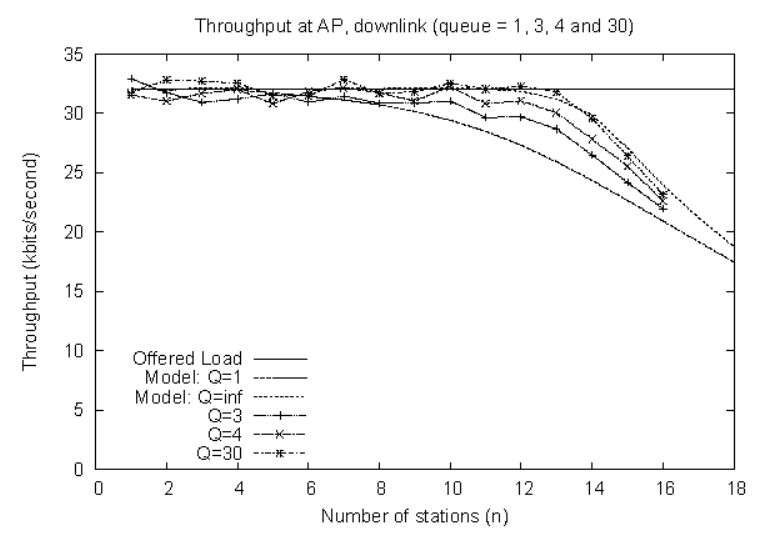

Fig. 2. Measured and modelled throughput at the AP for standard 802.11b MAC settings. Results are shown for various buffer sizes. The buffer size at the AP and the STAs is the same. Predictions using analytic modeling for a buffer size of 1 and an infinite buffer are shown and bound achieved throughput.

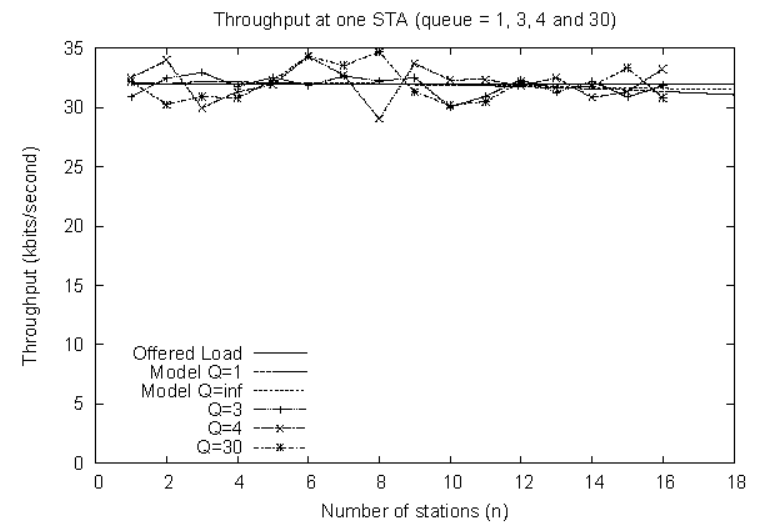

Fig. 3. Measured and modelled throughput at a single STA for standard $802.11 \mathrm{~b}$ MAC settings. The buffer size at the AP and the STAs is the same. Results are shown for various buffer sizes.

single source and does not have to deal with short-term buffering of packets from multiple sources.

To demonstrate that there is an asymmetry in buffering requirements, Figure 4 shows resulting AP throughput where the buffering at the AP and the stations is not the same. Figure 5 shows the corresponding STA throughput. From this we see that though buffer size affects throughput at the AP, STA throughput is insensitive to buffer size, a buffer of 4 packets is essentially sufficient at the stations.

Figure 4 also shows that the performance at the AP is quite similar for 15,30 and 399 packet buffers, in contrast to a 4 packet buffer where throughput drops by almost $10 \%$ at 10 nodes. This is in broad agreement with the predictions of the infinite buffer model shown in Figure 2. We believe this is because the main purpose of buffering at the AP is accommodate the multiplexing of sources, and that once we have buffering proportional to $n$ at the AP, there is little advantage to adding more. In the remainder of the paper, for simplicity, we will set the buffer at the STA and the AP to be the same. However we expect the STA's requirements to be much smaller.

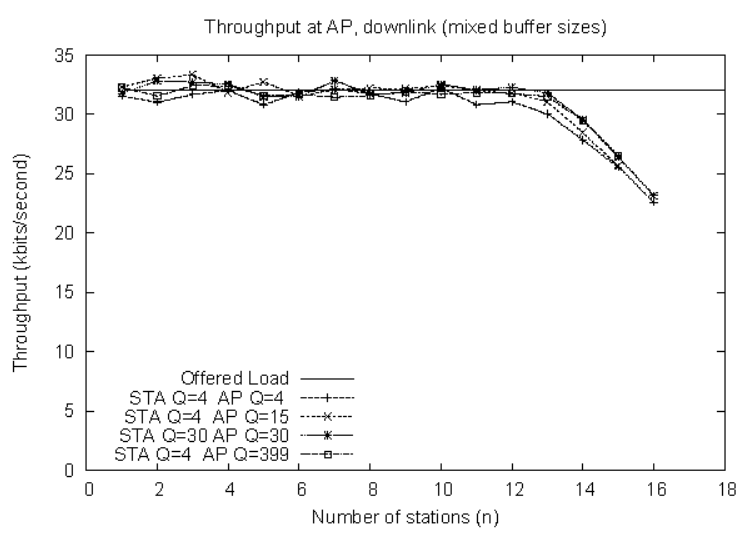

Fig. 4. Measured throughput at the AP for standard 802.11b MAC settings. Results are shown for various combinations of buffer sizes at the STAs and the AP.

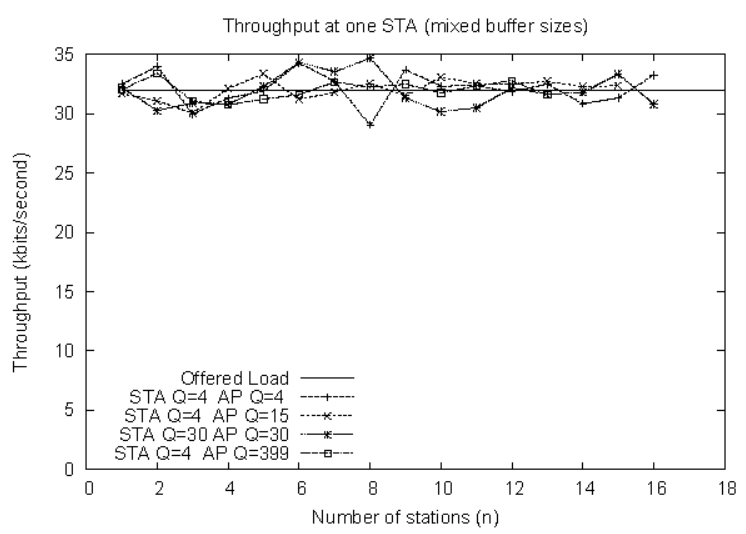

Fig. 5. Measured throughput at a single STA for standard $802.11 \mathrm{~b}$ MAC settings. Results are shown for various combinations of buffer sizes at the STAs and the AP.

Voice is sensitive to both throughput and delay performance. Figure 6 shows the cumulative distributions for MAC delay at the access point for a buffer size of 4 (too small for the AP) and 30 (OK for the AP). We show the delay distribution for three different numbers of STAs: 5 (underloaded), 10 (close to saturation) and 15 (overloaded). The delay shows some basic features such as an initial step, representing a minimum transmission time, and slight kinks, representing numbers of backoff stages/other STA's transmissions before the final successful transmission.

As we expect, in the underloaded situation the AP buffer size makes little difference to the delay distribution. Even for 10 stations, increasing buffering has little impact, which is surprising as increased buffering has a notable impact on throughput (cf. Figure 2). When overloaded, buffering has a small impact, slightly increasing the delay. We believe this is because when the network is busy, extra buffering allows more packets to reach the MAC layer for transmission. Each packet must contend for access and this reduces delay performance.

Figure 7 shows the corresponding delay distribution at a station. While increasing buffering only had a small impact on delay for the AP, the increase is slightly larger for the 


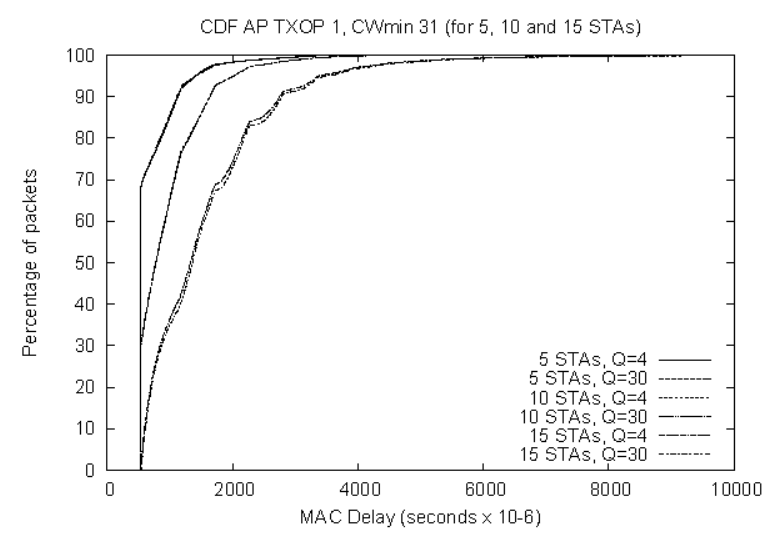

Fig. 6. Cumulative distribution for delays at the access point when there are 5,10 and 15 stations with standard 802.11b MAC settings.

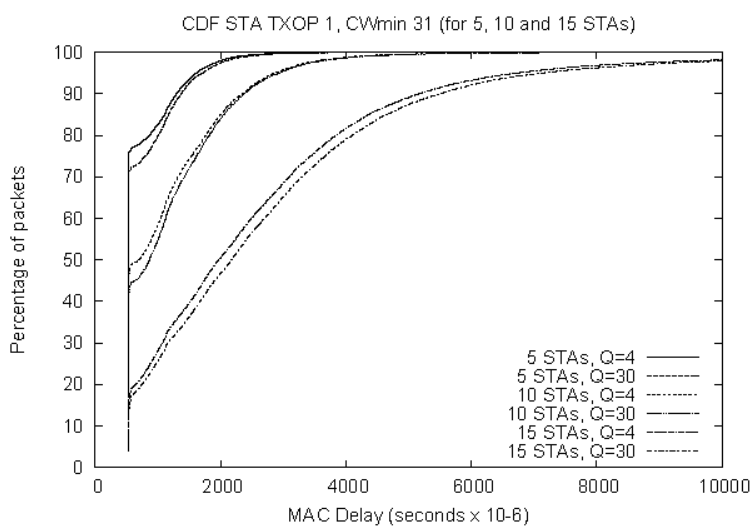

Fig. 7. Cumulative distribution for delays at a station when there are 5, 10 and 15 stations with standard $802.11 \mathrm{~b}$ MAC settings.

STA, resulting in a shift of a few tenths of a millisecond. This may be due to a combination of factors, for example we know increased buffering results in increased throughput at the $\mathrm{AP}$ and a consequent reduction in free time on the medium should result in increased transmission times for stations.

\section{Combined Impact of PRioritisation And BUFFERING}

By restoring parity in transmission opportunities between forward and reverse traffic in infrastructure networks the potential exists to increase voice call capacity. We consider prioritising the AP to restore parity between upstream and downstream flows. In the most simple case, we use the TXOP packet bursting mechanism to increase the number of packets that the AP can send at each transmission opportunity to be $n_{d}$, the number of downstream voice flows. We also consider the scheme where TXOP allows the AP to send $n_{d} / 2$ frames, but we also half the value of $C W_{\text {min }}$ to approximately double the number of transmit chances available to the AP. The aim of this is to see if frequent but smaller bursts have a more favourable impact on the delays of stations in the system.

Figure 8 and Figure 9 respectively show the throughput

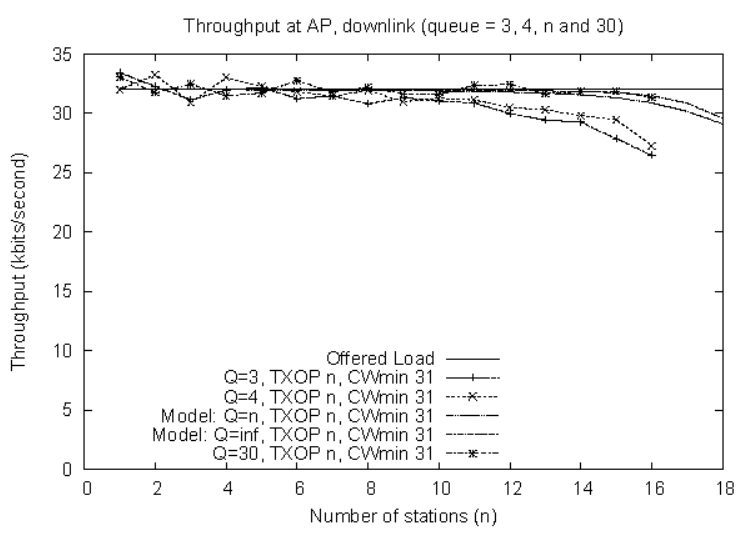

Fig. 8. Throughput at the AP for prioritised voice, with $T X O P=n$ packets. Results are shown for various experimental and modelled buffer sizes.

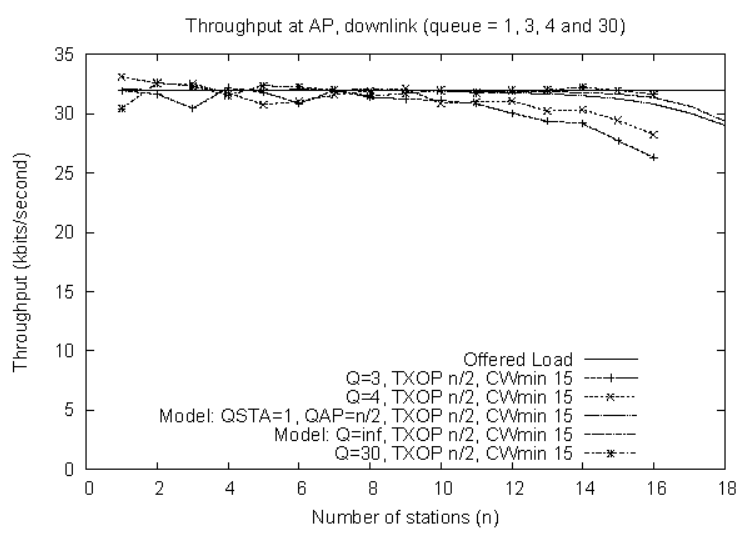

Fig. 9. Throughput at the AP for prioritised voice, with $T X O P=$ $n / 2$ packets, $C W_{\min }=15$. Results are shown for various experimental and modelled buffer sizes.

using these prioritised schemes. We see that 3 and 4 packet buffers are still insufficient, but for 30 packet buffers we can maintain throughput well above $90 \%$ of average load of $32 \mathrm{Kbps}$ out as far as 16 voice calls in the network. The models we use can model an infinite buffer or a buffer of $n$ packets. We see that these models predict almost the same throughput at a level consistent with our 30 packet buffer measurements. The throughput performance of the two schemes is similar. Intuitively we might expect this, as giving the AP a transmission of half the size twice as frequently should be similar as long as there is sufficient buffering to accommodate the bursts.

Regardless of buffering or prioritisation at the AP, throughput at our measurement station remained satisfactory for the range of STAs that we explored, as shown in Figure 10 (the other scheme shows similar performance). As shown, this is consistent with the predictions of the analytic models.

Figure 11 shows the mean MAC delay of packets at the access point. As the MAC delay corresponds to the service time of the queue, we require the mean service time to be less than the mean packet inter-arrival time. As expected, we see that prioritising the AP decreases the mean delay. 


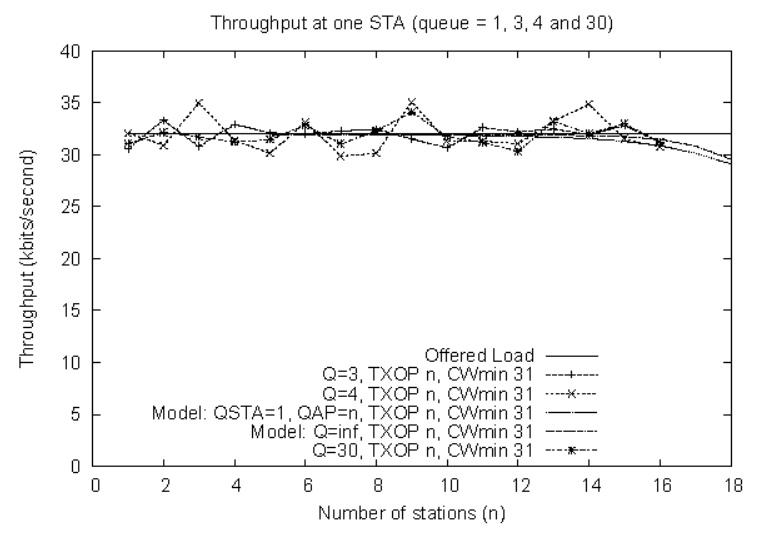

Fig. 10. Throughput at a STA for prioritised voice, with $T X O P=n$ packets at the AP. Results are shown for various buffer sizes.

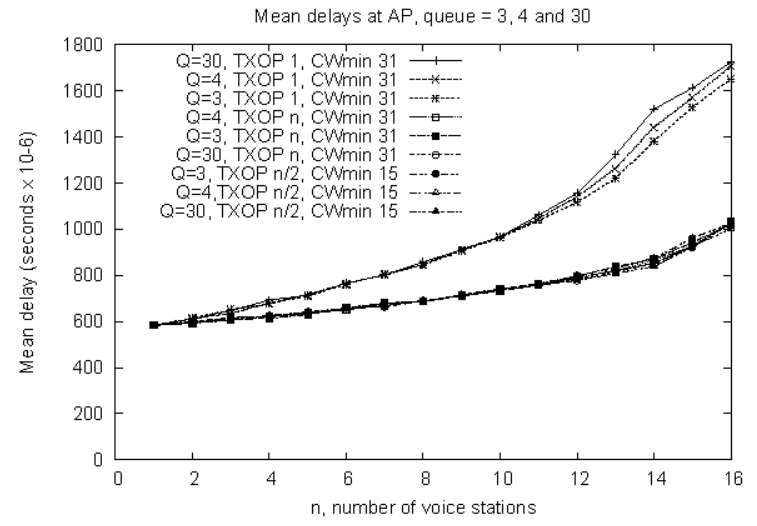

Fig. 11. Mean MAC delay at the AP for standard 802.11b MAC settings and when the access point is prioritised using $802.11 \mathrm{e}$. Results are shown for various buffer sizes. The group with longer mean delays correspond to the experiments in which the AP is not prioritised.

We can see that the impact of the queueing is largely insignificant. There is a small impact: without prioritisation we see that increasing the queue size increases the MAC delay, and so decreases the service rate. This is because increased buffering is leading to increased contention. Conversely, increasing the queue size in the prioritised case actually slightly lowers the mean service time. We believe this is because it increases the sizes of the bursts that the access point sends and so increases efficency without increasing contention.

Figure 12 shows that the stations' delay increases more rapidly when the $\mathrm{AP}$ is prioritised and the the number of stations is more than 14. Examining the performance for 16 stations reveals three groups: those where the AP is unprioritised $(\approx 3000 \mathrm{~ms}$ delay $)$, those where the AP is prioritised but has a small buffer $(\approx 4000 \mathrm{~ms}$ delay $)$ and those where the AP is prioritised with a large buffer $(\approx 7000 \mathrm{~ms}$ delay). We have seen that to maintain AP throughput we need both a larger buffer and prioritisation, so we conclude that we are nearing the boundary of the stability region for STAs. The exact prioritisation used does not have a significant impact on the stations' mean delay. This is inter-

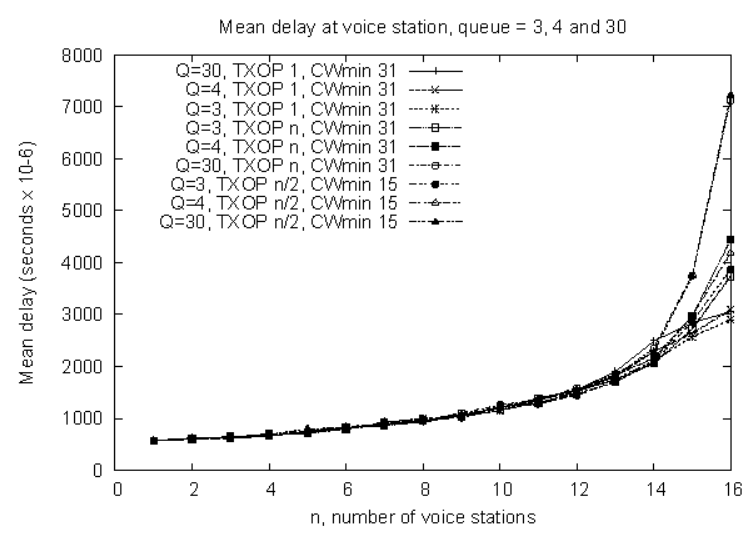

Fig. 12. Mean MAC delay a station for standard 802.11b MAC settings and when the access point is prioritised using $802.11 \mathrm{e}$. The mean inter-packet arrival time at a STA is $10000 \mu s$.

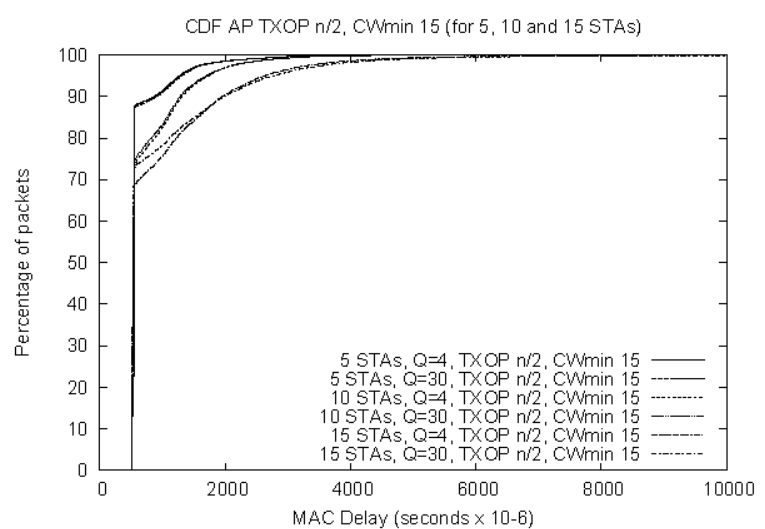

Fig. 13. Cumulative distribution for delays at the access point when there are 5, 10 and 15 stations when the AP is prioritised using $T X O P=n / 2$ packets and $C W_{\min }=15$.

esting, as one might expect longer bursts to lead to longer mean delays.

Figure 13 shows the delay cumulative distributions at the access point when using $T X O P=n / 2$ packets and $C W_{\min }=15$. The results for $T X O P=n$ are similar. Compare Figure 6 and Figure 13. Naturally, prioritising the AP has improved the delay distribution in all cases.

However, the impact of buffering is now slightly different to the unprioritised case (Figure 6). Again, when the system is below saturation we see buffering having little impact. However, when the system is overloaded, in the prioritised situation increased buffering actually makes the delay distribution marginally better. This may be due to increased buffering allowing longer TXOP bursts, and this can increase the rate at which packets are cleared without significantly increasing the amount of contention on the network.

The use of TXOP at the AP may also have an impact on the stations. A large burst of packets transmitted by the AP might have a detrimental effect on the jitter experienced by a station's packet. As bursts are smaller in the prioritised scheme with $T X O P=n / 2$ packets and 


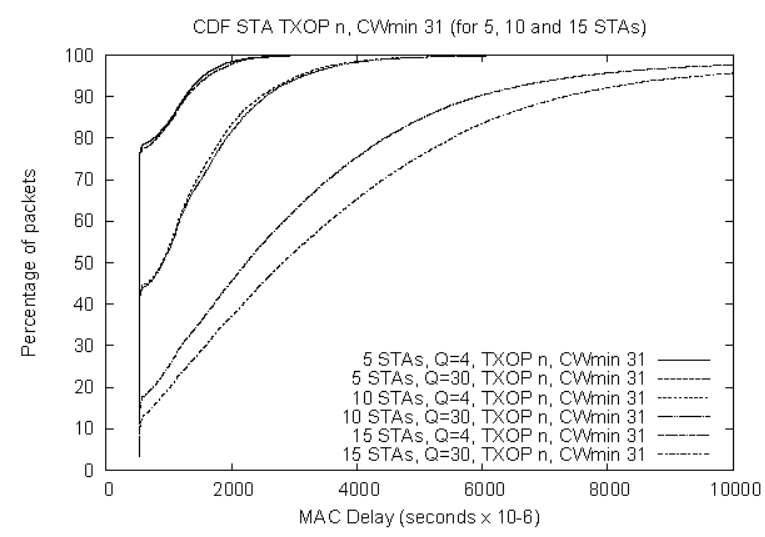

Fig. 14. Cumulative distribution for delays at a station when there are 5,10 and 15 stations when the $\mathrm{AP}$ is prioritised using $T X O P=n$ packets.

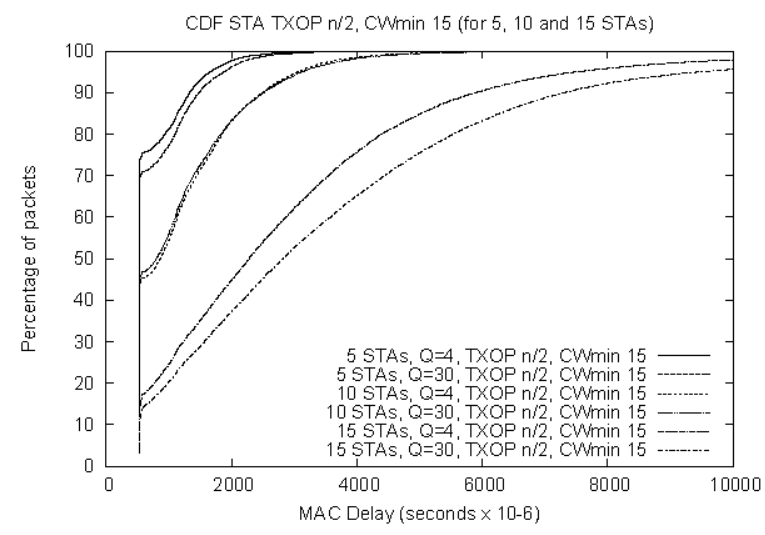

Fig. 15. Cumulative distribution for delays at a station when there are 5,10 and 15 stations when the AP is prioritised using TXOP= $n / 2$ packets and $C W_{\min }=15$.

$C W_{\min }=15$, we might expect it to be more kind to the STA. Figure 14 shows the delay distribution at a STA when the access point has long bursts given by $T X O P=n$ packets. Compared to Figure 7 there is little change, perhaps a marginal worsening of the delay distribution, however it seems to be limited to a few tenths of a millisecond in below-saturation cases. Figure 15 shows the results when the AP is prioritised with smaller, but more frequent bursts. While there again are some small differences, for the underloaded and below-saturation examples there are no substantial differences between the scheme.

Note that increased buffering in the system uniformly results in no improvement the STA's delay distribution, regardless of the prioritisation or load in the system. This is in contrast to the situation at the AP where the impact of buffering depended on the presence of prioritisation. This is in line with our observations that little buffering is sufficient for an individual STA, and increased buffering at the AP may hinder a STA's performance.

\section{Conclusion}

We have demonstrated that throughput at the AP is the main constraint on capacity for voice traffic in infrastructure mode 802.11 networks, (e.g., reducing the capacity to less than 10 calls), confirming previous analytic and simulation based studies. We show that buffering can partially alleviate this problem (e.g., allowing up to 12 calls). We note that the buffering requirements at the access point are different from those at a station; while a station requires approximately fixed buffering the access point requires buffering proportional to the number of calls. TXOP and $C W_{\min }$ are shown to be effective in further resolving this problem (e.g., increasing the capacity to around 16 calls). The impact on delay and jitter introduced by $T X O P$ does not seem to pose a barrier to its use. We have also confirmed a subtle interaction between buffering and contention which can be observed by measuring MAC service times, which may indicate a small advantage to using smaller buffers for delay sensitive traffic.

\section{REFERENCES}

[1] A. Ng, D. Malone, and D. Leith, "Experimental evaluation of TCP performance and fairness in an 802.11 e test-bed," in $A C M$ SIGCOMM Workshops, 2005.

[2] Q. Ni, "ns-802.11e EDCF for IEEE 802.11e wireless LAN," http: //www-sop.inria.fr/planete/qni/, INRIA, Tech. Rep., November 2002.

[3] S. Wiethölter and C. Hoene, "Design and verification of an IEEE 802.11e EDCF simulation model in ns-2.26," Technische Universität Berlin, Tech. Rep. TKN-03-019, November 2003.

[4] G. Bianchi, "Performance analysis of IEEE 802.11 distributed coordination function," IEEE Journal on Selected Areas in Communications, vol. 18, no. 3, pp. 535-547, March 2000.

[5] R. Battiti and B. Li, "Supporting service differentiation with enhancements of the IEEE 802.11 MAC protocol: models and analysis," University of Trento, Tech. Rep. DIT-03-024, May 2003.

[6] E. Pelletta and H. Velayos, "Performance measurements of the saturation throughput in IEEE 802.11 access points," in WiOpt, April 2005.

[7] M. Franceschinis, M. Mellia, M. Meo, and M. Munafo, "Measuring TCP over WiFi: A real case," in WiNMee, April 2005.

[8] I. Dangerfield, D. Malone, and D. Leith, "Experimental evaluation of $802.11 \mathrm{e}$ EDCA for enhanced voice over WLAN performance," in International Workshop On Wireless Network Measurement (WiNMee), 2006.

[9] N. Hegde, A. Proutiere, and J. Roberts, "Evaluating the voice capacity of 802.11 WLAN under distributed control," in Proc. IEEE LANMAN, September 2005.

[10] P. Clifford, K. Duffy, D. Leith, and D. Malone, "On improving voice capaicty in 802.11 infrastructure networks," in Proc. IEEE WirelessCom, June 2005

[11] D. Malone, P. Clifford, and D. Leith, "On buffer sizing for voice in 802.11 WLANs," IEEE Communications Letters, vol. 10, no. 10 , October 2006.

[12] K. Duffy and A. Ganesh, "Modeling the impact of buffering on 802.11," IEEE Communications Letters.

[13] "Soekris engineering," http://www.soekris.com/.

[14] "Multiband Atheros driver for WiFi (MADWiFi)," http:// sourceforge.net/projects/madwif/, r1645 version.

[15] V. Jacobson, C. Leres, and S. McCanne, "tcpdump," http:// www.tcpdump.org/.

[16] A. Markopoulou, F. Tobagi, and M. Karam, "Assessing the quality of voice communications over internet backbones," IEEE Transactions on Networking, vol. 11, no. 5, pp. 747-760, October 2003.

[17] K. Duffy, D. Malone, and D. Leith, "Modeling the 802.11 Distributed Coordination Function in non-saturated conditions," IEEE Communications Letters, vol. 9, no. 8, pp. 715-717, 2005. 